\section{A propósito de una experiencia de extensión universitaria en promoción de salud desde la Psicología en el ámbito educativo}

\author{
Déborah Rydel \\ Universidad de la República, Uruguay. \\ deboryd12@gmail.com \\ (iD) orcid.org/0000-0003-3230-7595
}

RECEPCIÓN: 28/05/20

ACEPTACIÓN FINAL: 13/08/20

\section{Resumen}

Este artículo relata y analiza una experiencia de trabajo desarrollada durante casi una década en el Primer Nivel de Atención en Salud, en una escuela pública en el departamento de Canelones, Uruguay. La experiencia forma parte de una práctica integral donde participan estudiantes avanzados de la Facultad de Psicología de la Universidad de la República y actores e instituciones comunitarias. El objetivo de esta presentación es realizar aportes teóricos y metodológicos para la formación de los estudiantes de Psicología en el ámbito del Primer Nivel de Atención en Salud así como contribuir a la difusión de experiencias en extensión universitaria que pueda servir como referencia para intervenciones similares en contextos institucionales y socioculturales diversos.

Palabras clave: práctica integral; primer nivel de atención en salud; psicología; escuela pública; extensión universitaria.
About a university extension experience in Health Promotion from Psychology in the public school

\section{Abstract}

This article relates and analyzes a work experience developed during almost a decade in the Primary Health Care. The experience is part of a comprehensive practice involving advanced students from the UdelaR School of Psychology and community actors and institutions. The objective of the same, is to contribute to the diffusion of valuable experiences in university extension for its recreation in diverse institutional and sociocultural contexts. Also contribute to theoretical reflection and construction of methodological knowledge regarding the PNA, as well as healthy coexistence in the school environment.

Keywords: comprehensive practice; Primary Health Care; psychology; educational field. training of human resources for work in the
Sujetos y relaciones en extensión universitaria /
Acerca de uma experiência de extensão universitária em promoção da saúde a partir da Psicologia no âmbito educacional

\section{Resumo}

Este artigo relata e analisa uma experiência de trabalho desenvolvida durante quase uma década no Nível Primário de Atenção à Saúde. A experiência faz parte de uma prática abrangente que envolve estudantes avançados da Escola de Psicologia de UdelaR e atores e instituições da comunidade. O objetivo do mesmo é contribuir para a difusão de experiências valiosas na extensão universitária para sua recreação em diversos contextos institucionais e socioculturais. Contribuem também para a reflexão teórica e construção de conhecimentos metodológicos sobre a formação de recursos humanos para o trabalho no Nível Primário de Atenção à Saúde, bem como a convivência saudável no ambiente escolar.

Palavras-chave: prática abrangente; níve primário de atenção; psicologia; ambiente educacional.

Para citación de este artículo: Rydel, D. (2020). A propósito de una experiencia de extensión universitaria en promoción de salud desde la Psicología en el ámbito educativo. +E: Revista de Extensión Universitaria, 10(13), e0021. doi: 10.14409/extension.2020.13.Jul-Dic.e0021 


\section{Introducción}

La experiencia que se presenta aquí forma parte de una práctica compleja cuyo nombre inicial fue "Construyendo modelos de intervención desde la Psicología de la Salud" y que se realizó desde el año 2003 hasta 2018. La misma se propuso desplegar modelos de intervención propios del psicólogo en el Primer Nivel de Atención (PNA) con el objeto de contribuir a la ampliación de acciones que redundaran en mejorar la calidad de atención con énfasis en la promoción y prevención en salud. Se llevó a cabo con estudiantes avanzados en la carrera de Psicología, en diversas zonas de Montevideo y su área metropolitana. En la misma se articularon los cometidos de la Universidad y las prácticas integrales ${ }^{1}$ desarrolladas en el marco de la Segunda Reforma Universitaria, ${ }^{2}$ con el cambio de modelo propuesto por el Sistema Nacional Integrado de Salud (SNIS). ${ }^{3}$ Se jerarquizó el abordaje integral e interdisciplinario en el contexto de la estrategia de Atención Primaria de la Salud (APS) y se conjugó la formación de estudiantes para el PNA y el trabajo en extensión universitaria integrando actores e instituciones diversas.

Esta práctica se ha ido transformando con el transcurso del tiempo, ha pasado por etapas de crecimiento y complejidad. Durante todos estos años hubo grandes reformas además de la producida en el ámbito de salud con la creación SNIS, desde los realizados en la estructura académica hasta en el Plan de Estudios de la Licenciatura de la Facultad de Psicología; cambios que impactaron en los equipos docentes, en los estudiantes y, por lo tanto, en las prácticas. También hubo transformaciones en las concepciones teóricas, en las intervenciones que desde la Psicología se plantean en el PNA, así como en los barrios, en las instituciones con las que se trabaja y en la sociedad en su conjunto.

A lo largo de este recorrido, la preocupación de las docentes por reconstruir, dar a conocer y mejorar la intervención en sus diversos aspectos fue una constante. Así, las intervenciones efectuadas fueron cambiando de forma, de lugares, de actores, incorporando nuevas estrategias y dispositivos de trabajo. Finalmente, la práctica debió ser reestructurada para ser llevada adelante solamente por la autora de este artículo. ${ }^{4}$ Este cambio implicó una

1) Las prácticas integrales se definen por la integración de los procesos de enseñanza, investigación y extensión, con una perspectiva interdisciplinaria y un enfoque territorial e intersectorial en sus abordajes. Tienen intencionalidad transformadora, conciben a los actores sociales como sujetos protagonistas de dichas transformaciones y no como objeto de intervenciones universitarias. El foco del aprendizaje se pone en los procesos y en el diálogo entre personas de formaciones diversas, universitarios y no universitarios, que abordan un problema de interés común.

2) Proceso de cambios profundos, iniciado en 2007, bajo el rectorado de Rodrigo Arocena (2006-2014), en el cual se apuntaba a la democratización del conocimiento a través de una educación universitaria pública para todos. Se trataba de posibilitar que las mayorías accedieran a formas de la enseñanza superior y permanente relacionada con el mundo del trabajo, la cultura y la mejora de la calidad de vida individual y colectiva. Para ello se adoptaron decisiones para la expansión de las actividades de enseñanza, investigación y extensión en todo el país, cooperando con la sociedad en la solución de problemas sociales y en la mejora de la producción.

3) La reforma del Sistema de Salud fue presentada y llevada a cabo por el Frente Amplio, partido político que en 2005 alcanzó por primera vez el gobierno nacional. Su implementación fue un proceso secuencial que dio lugar a la creación del Sistema Nacional Integrado de Salud (SNIS) (Ley 18.211, 2007), al Seguro Nacional de Salud (SNS) y al Fondo Nacional de Salud (FONASA) en 2008. A grandes rasgos, la reforma planteó cambios en tres niveles: el modelo de financiamiento, asegurando cobertura universal, equidad y solidaridad a través del SNS y el FONASA; el modelo de gestión, democratizándola, haciéndola más eficiente y garantizando su transparencia; y el modelo de atención orientado a la aplicación de una estrategia de Atención Primaria de la Salud (APS), asegurando la mayor capacidad resolutiva del primer nivel de atención.

4) El equipo docente esta integrado inicialmente con las Prof. agregada Adriana Gandolfi y la asistente Mónica Cortázar. 
focalización de la intervención en la zona de Empalme Nicolich, en el número de estudiantes que participan, y luego en las instituciones con las que se trabaja.

Cada año, después de analizar las necesidades y los pedidos relevados en el diagnóstico de salud de la zona con los referentes comunitarios y de evaluar nuestras posibilidades, se hacía la selección de las instituciones o actividades y dónde o con quiénes se iba a trabajar. Todo ello de carácter muy variado, como por ejemplo la Policlínica de salud pública de la Administración de Servicios de Salud del Estado (ASSE) con distintas temáticas y grupos etarios, instituciones educativas (Caif, ${ }^{5}$ jardín de infantes, escuela, liceo), radios comunitarias, y organizaciones que se daban los vecinos para llevar a cabo alguna tarea para el barrio.

En los últimos años se mantiene el trabajo en la escuela de la zona y con adultos mayores en coordinación con la Policlínica de ASSE. ${ }^{6}$

El objetivo de este artículo es contribuir a la difusión de una experiencia de salud y de convivencia escolar y que pueda servir como referencia para otros trabajos similares en contextos institucionales y socioculturales diversos. Por lo cual aquí se recupera y analiza el trabajo en la escuela pública de Empalme Nicolich en el departamento de Canelones. En particular, los talleres de convivencia saludable realizados con grupos escolares de 4 to. y 5to. años entre los años 2011 y 2017. Se aspira a aportar a la reflexión teórica y construcción de conocimiento metodológico respecto de la convivencia saludable en el ámbito escolar, así como a la formación de recursos humanos para el trabajo en el PNA.

Resulta pertinente en esta convocatoria de $+E$ mostrar la posibilidad de articular en un territorio determinado la formación integral de estudiantes universitarios con demandas de la comunidad, con redes de APS e instituciones educativas. Los estudiantes acceden a experiencias preprofesionales desde el intercambio con otros actores (equipo docente, equipo de salud, referentes comunitarios, niños) y contribuyen a las transformaciones sociales.

Se propone al lector ir conociendo la localidad de Empalme Nicolich, la Escuela $N^{\circ} 171$ Dinamarca y los aportes teóricos que sustentaron la intervención. Luego se describe la metodología de trabajo en toda su complejidad y la actividad en los talleres para finalizar con el análisis crítico de la experiencia realizada.

\section{El escenario}

Empalme Nicolich (Santa Teresita) es una de las localidades que conforman el Municipio Nicolich desde el año 2010 junto a Villa Aeroparque, Colonia Nicolich y cuatro barrios privados (Altos de la Tahona, La Asunción, Quintas del Bosque, Colinas de Carrasco), dentro del área metropolitana en el departamento de Canelones. Está atravesada por las rutas nacionales 101 y 102 y se encuentra en las cercanías del Aeropuerto Internacional de Carrasco. A partir de mayo de 2017 la zona fue declarada Ciudad Liber Seregni, en reconocimiento a su crecimiento urbano y poblacional. Cuenta con una población aproximada de 14800 habitantes, siendo Empalme Nicolich la localidad más poblada. Es el municipio con mayor porcentaje de jóvenes del departamento. En el año 2010 había un 52 \% de sus habitantes con ne-

5) Centros de Atención a la Infancia y la Familia.

6) La coordinación general de la intervención de promoción de salud en la zona es llevada a cabo por la autora de este trabajo en coordinación con los referentes institucionales. 
cesidades básicas insatisfechas. Al crearse, las autoridades municipales convocaron a la comunidad, a las instituciones públicas, a empresarios y al gobierno nacional para diseñar el Plan de Desarrollo y Ordenamiento Territorial de la zona con el objetivo de favorecer el acercamiento de programas y políticas de múltiples organismos e instituciones del Estado, además de proporcionar espacios de participación y apropiación ciudadana. De esta manera, en los últimos años fueron llegando servicios según lo demandó el crecimiento poblacional y el proceso de urbanización, que cambiaron la geografía y el funcionamiento de la zona. En palabras de los vecinos y referentes en entrevistas realizadas en el marco del diagnóstico de situación comunitario:

"antes era todo más precario, era como que no había nada de nada, si le preguntabas a los niños si conocían el aeropuerto decían que no, no sé dónde queda... ha cambiado la movilidad en el barrio, antes veías como mucho un carro con un caballo, ahora ves motos.... ipasan ómnibus!".

Una estudiante se refiere al lugar como de "características propias de un pueblo, los pobladores tienen contacto directo con los servicios y los funcionarios... con nosotros se mostraron muy abiertos".

Conforme a los datos recabados de los diagnósticos de situación comunitaria realizados en la zona, los problemas más relevantes de la comunidad fueron variando. Inicialmente estaban relacionados con la salud y el trabajo y luego predominaron los de educación, vivienda y seguridad vial. Otras problemáticas recogidas fueron: dificultades económicas, violencia, alcoholismo, drogas y analfabetismo.

Para este artículo se seleccionó la experiencia que hemos llamado "Convivencia saludable en el ámbito educativo" realizada durante siete años consecutivos en la Escuela Pública № 171, Dinamarca. La tarea que se llevó a cabo en la escuela fue coordinada año a año con la maestra de apoyo en acuerdo con el Equipo de Dirección de la escuela. La misma incluyó diversas modalidades de intervención, entre ellas los talleres con niños y niñas.

La Escuela Dinamarca es una de las tantas escuelas del país consideradas de contexto sociocultural crítico y está comprendida dentro del Programa de Escuelas APRENDER (Atención Prioritaria en Entornos con Dificultades Estructurales Relativas). Este Programa se creó en el año 2011 y responde a orientaciones de una política educativa que reconoce la necesidad de integrar y articular acciones que se focalicen en los sectores más vulnerables.

Según el consejero Héctor Florit (Consejo de Educación Inicial y Primaria, CEIP, 2014), se procura atender a las escuelas:

"en función de las características socioculturales de la comunidad a la que pertenecen sus alumnos, refleja la necesidad de entender el contexto para poder trabajar a partir de él, de su conocimiento, del respeto por la diversidad y de la gestión participativa donde la presentación de proyectos fomenta el protagonismo de todos los actores". (p.1)

La escuela

"se construye desde una concepción de la educación como derecho humano fundamental pleno, que supone su desarrollo en sintonía con el tiempo, las realidades y las necesidades de aprendizaje de los niños y las niñas en cada contexto y momento". (Terigi, 2009, p.17) 
Debe ser forjadora de ciudadanía democrática y construir normas de convivencia a partir de la vivencia de valores como la libertad, el pluralismo, la justicia, la solidaridad, la tolerancia, la autoestima y el respeto (CEIP, Programa APRENDER).

En los últimos años, el número de niños y niñas que asisten a la Escuela $N^{\circ} 171$ fue decreciendo debido a la creación de nuevas escuelas en la zona, pero mantuvo la dotación docente, por lo cual mejoraron en ese sentido las condiciones de trabajo, ya que los grupos tienen en promedio 20 alumnos. Vemos que los docentes organizan el trabajo de acuerdo con diferentes estrategias y/o formatos, ya que se constatan niveles de rendimientos muy descendidos, altos niveles de repetición y ausentismo. Por la misma razón, la escuela cuenta con recursos complementarios, como maestras comunitarias, ${ }^{7}$ maestra de apoyo, ${ }^{8}$ profesores de gimnasia y, en algún año, danza. Posee un comedor que funciona para los dos turnos de la escuela donde se prepara la comida y se brinda una merienda.

Las familias de los niños que concurren a la escuela, en general, son de muy escasos recursos, predominan los hogares monoparentales debido a abandonos o muertes, y madres con varios hijos sin trabajo remunerado. Al decir de una maestra: "Se viven situaciones muy duras: necesidades básicas insatisfechas, bajo nivel de alfabetización, núcleos familiares desarticulados, niños con responsabilidades de adultos y que en ocasiones no cuentan con ningún tipo de apoyo".

Otra maestra refiere que son "madres muy jóvenes, con embarazos no controlados, con medicación o abuso de sustancias... eso deja secuelas a nivel orgánico". Debido a la falta de recursos, "la inasistencia de los niños cuando llueve es muy alta, no tienen vestimenta para cambiarse si se mojan", comenta otra maestra. Actualmente, a raíz de las mejoras en la institución escolar y la menor cantidad de niños que asisten, "es preferible venir a clase que quedarse arriba de las camas cuando sus casas se inundan", dice otra maestra.

Las principales preocupaciones que plantea el equipo de dirección y los y las docentes giran en torno a: dificultades de aprendizaje, problemas de conducta y de relacionamiento, dificultades en el cuidado de las instalaciones y materiales, y sobrecarga al rol tradicional del maestro o de la maestra. La escuela participa, al igual que la mayor parte de las instituciones y programas que operan en el zona, de las redes y de los Nodos Interinstitucionales (Educación, No a la Violencia) convocados por el Servicio de Orientación, Consulta y Articulación Territorial -SOCAT—). ${ }^{9}$ Estos servicios apuntan a impulsar el desarrollo comunitario y la activación de redes de protección local a través de la participación de vecinos e instituciones públicas y privadas que tienen en común el hecho de trabajar o vivir en el mismo territorio.

7) Programa cuyo objetivo es mejorar la calidad de los aprendizajes de los niños sobre la base de los vínculos de la escuela con las familias y de encontrar pedagógica y didácticamente los caminos para restituir el deseo de aprender perdido por diversas causas. Tiene cuatro líneas de acción: alfabetización en hogares, grupos con las familias, integración educativa y aceleración escolar.

8) Figura creada en la escuela común a partir de una reestructura integracionista en Educación Especial (1985) con el propósito de favorecer la integración escolar de niños y niñas con problemas para aprender y discapacidad.

9) EI SOCAT es un servicio que apunta a impulsar el desarrollo comunitario y la activación de redes de protección local a través de la participación de vecinos e instituciones públicas y privadas que tienen en común el hecho de trabajar o vivir en el mismo territorio. Los problemas detectados requieren en general de la acción articulada de varias instituciones para poder ser resueltos, lo que da lugar a la conformación de espacios de articulación territorial como los Nodos, donde convergen técnicos de diversos organismos a fin de complementar y mejorar la eficacia de las acciones a través de un quehacer en común. 
Son implementados por organizaciones de la sociedad civil, en convenio con el Ministerio de Desarrollo Social (MiDeS). Los Nodos se reúnen mensualmente, pero frente a alguna situación puntual que requiere pensar una estrategia de abordaje en menor tiempo se convocan las redes focales.

\section{Fundamento teórico}

La APS, rectora de los cambios de modelos de atención del SNIS constituye una estrategia global que se liga naturalmente al concepto de atención integral. Para dar cuenta de ella se hace imprescindible el fortalecimiento del PNA como un objetivo relevante. Desde esta estrategia se concibe a la salud como un derecho humano fundamental a ser ejercido por la población, que estimule el desarrollo de la participación local, promueva acciones comunitarias e intersectoriales mediante la coordinación y realización de proyectos conjuntos con instituciones educativas, vecinos, ONG, y que impulse la construcción de redes. El PNA es el primer nivel de contacto de los individuos, la familia y la comunidad con el sistema de salud y lleva lo más cerca posible la atención al lugar donde residen y trabajan las personas (Calatayud, 2009). En el PNA la posibilidad de incidir en los determinantes sociales de la salud es mayor así como la capacidad de generar espacios de participación comunitaria dirigidos a profundizar la participación de las organizaciones de la sociedad en los ámbitos de toma de decisión y evaluación.

La promoción de salud, tal como se enuncia en la $1^{\circ}$ Conferencia Internacional sobre la Promoción de Salud: Carta de Ottawa (1986), consiste en "proporcionar a los pueblos los medios necesarios para mejorar su salud y ejercer un mayor control sobre la misma. (...) Se trata de un concepto positivo que acentúa los recursos sociales y personales así como las aptitudes físicas" (p. 1). La Carta señala cinco áreas de acción para la promoción de salud: formulación de políticas públicas para la salud, creación de entornos saludables, desarrollo de aptitudes personales para la salud, fortalecimiento de la acción comunitaria y reorientación de los servicios de salud.

"La salud se crea y se vive en el marco de la vida cotidiana: en los centros de enseñanza, de trabajo y de recreo. La salud es el resultado de los cuidados que uno se dispensa a sí mismo y a los demás, de la capacidad de tomar decisiones y controlar la vida propia y de asegurar que la sociedad en que uno vive ofrezca a todos sus miembros la posibilidad de gozar de un buen estado de salud". (p. 1)

Uno de sus instrumentos fundamentales es la educación para la salud que, según la OMS, debe ser concebida como un "proceso que promueve cambios de conceptos, comportamientos y actitudes frente a la salud, a las enfermedades y al uso de servicios, que refuerza conductas positivas" (1999, p. 20). Educar en salud tiene como objetivos principales permitir a las personas definir sus propios problemas y necesidades, comprender lo que pueden hacer sobre esos problemas con sus propios recursos combinados con el apoyo exterior y decidir cuál es la acción más apropiada para fomentar una vida sana y el bienestar de la comunidad. Desde esta perspectiva, compete a los profesionales de la salud promover y facilitar estos procesos en las comunidades, respetando sus saberes, a través del estímulo de la participación activa, hacia un mayor conocimiento y control de su bienestar. No es posible 
que el sistema de salud por sí solo pueda alcanzar las metas que se propone, se requiere de un esfuerzo coordinado de los distintos sectores sociales, donde es importante la iniciativa de los mismos a la vez que la receptividad a convocatorias de los otros sectores. Se presupone la articulación intersectorial, es decir,

"el trabajo coordinado de instituciones representativas de distintos sectores sociales, mediante intervenciones conjuntas destinadas a transformar la situación de salud y aportar al bienestar y calidad de vida de la población". (Organización Panamericana de la Salud —OPS—, 1999, p. 28)

La participación activa de las personas y comunidades es también parte inherente de la promoción de salud. Es necesario que las personas se asuman como sujetos activos que ejercen derechos, proponen, cuestionan y aportan a la comunidad para lograr un mayor empoderamiento. Y es fundamental aportar a la creación y mantenimiento de los espacios de participación locales y promover la apropiación de los mismos por parte de la comunidad.

El trabajo desde la promoción y prevención en salud en el ámbito educativo abre una amplia gama de posibilidades, como, por ejemplo, contribuir al mejoramiento de las condiciones de de enseñanza-aprendizaje, acompañar el proceso evolutivo de niños y niñas, y fortalecer el vínculo familia-institución-comunidad. En el ámbito escolar los niños adquieren conocimientos que van más allá de lo curricular y que se convierten en indispensables para su vida, lo cual permite el pasaje de un micromundo familiar a una posterior adaptación activa y creativa a la comunidad de la que forman parte. Podemos decir que el manejo de las emociones, su expresión de manera constructiva y la resolución de conflictos y situaciones de la vida cotidiana constituyen algunos de los principales conocimientos que se adquieren en esta etapa. Estas habilidades fomentarán en los y las jóvenes, los niños y las niñas, la autoestima y factores de protección para hacer frente a situaciones problemáticas. La escuela aporta a niños y niñas la posibilidad de aprender formas no violentas de resolver conflictos (Valdés y Klasse, 2008), por lo cual resulta de vital importancia contribuir y fomentar el buen trato y los vínculos saludables que contribuyan no solo a su sino también al buen funcionamiento institucional y social, entendiendo que estos aprendizajes pueden ser transmitidos y reproducidos en todos los ámbitos en que se desempeña la persona. La escuela es un agente de socialización esencial que para realizar su tarea adecuadamente tiene que poder reconocer sus problemáticas e incluirlas en sus objetivos educativos, aceptar que la familia, la sociedad y la infancia han ido cambiando y que esto significa cambiar las prácticas para una nueva realidad y forma de pensar la institución.

Los niños y niñas con los que se trabaja oscilan entre los 9 y 12 años de edad, con un promedio de 10. Desde el punto de vista cognitivo (Piaget, 1968) se encuentran en el estadio de las operaciones concretas, en el cual pueden comenzar a manejar conceptos aunque limitados en extensión y comprensión. Pueden descentrarse del punto de vista propio tomando distancia del egocentrismo característico del estadio anterior preoperatorio. Afectivamente, aparece una mayor regulación del comportamiento, los juegos reglados y el respeto y cooperación mutua entre pares. Los sentimientos morales comienzan a subordinarse a una escala permanente de valores. Según el desarrollo afectivo-sexual (Freud, 1924) se encuentran en el período de latencia. Lo específico de esta etapa es que la energía sexual, al menos gran parte de ella, es dirigida a un fin sublimatorio que logra establecer una nueva dinámica 
y reorganización de toda la estructura psíquica. Se modifica el decurso y destino pulsional y se da un refinamiento del sistema defensivo, con el consecuente control de la ansiedad y estabilización de la conducta. Se genera una creciente capacidad del yo para controlar las tendencias regresivas, una ampliación del mundo relacional así como de sus capacidades y actividades, con el consiguiente distanciamiento de las figuras parentales. Aumenta la capacidad simbólica de pensamiento y el lenguaje y su utilización para diversos aprendizajes, aparecen la autocrítica y una regulación de la autoestima más centrada en los logros y el consenso. Se observa una reducción del uso expresivo de todo el cuerpo, un aumento de la verbalización, un intento de dominar el medio ambiente a través del aprendizaje y una creciente autonomía. El papel del "otro" y de "los otros" es determinante para que se dé o no el "trabajo de latencia" (Urribarri, 1999) o para que se vea dificultado. Este autor habla de trabajo de latencia para señalar que no se trata de algo que viene determinado por la biología o por la herencia (Freud, 1924), sino de un proceso complejo que se dará o no con determinadas características en función de ciertas condiciones.

\section{El taller}

Desde hace algunos años, el concepto de taller se ha extendido de su uso corriente como el lugar donde se hace, construye o repara algo, al campo de la educación, con la idea de ser una metodología de trabajo grupal que promueve y jerarquiza la participación activa de los integrantes del grupo. Tiene como principal objetivo favorecer que los participantes expresen sus opiniones, dudas y certezas, y facilitar así la producción conjunta de nuevos saberes. González (1987) dice: "Me refiero al taller como tiempo-espacio para la vivencia, la reflexión y la conceptualización, como síntesis del pensar, el sentir y el hacer. Como el lugar para la participación y el aprendizaje" (p. 2). Ander Egg, (1991) lo define como "una modalidad pedagógica de aprender haciendo" (p. 10) tomando el principio de aprendizaje de Froebel (1826), para quien "aprender una cosa viéndola y haciéndola es algo mucho más formador, cultivador, vigorizante que aprenderla simplemente por comunicación verbal de las ideas". El taller así concebido permite superar la dicotomía entre la formación teórica y la experiencia práctica, superar el concepto de educación tradicional en el cual las y los estudiantes han sido receptores pasivos del conocimiento. Facilita el diálogo de saberes entre los participantes y la creación colectiva de los aprendizajes. Permite desarrollar actitudes reflexivas y críticas promoviendo espacios reales de comunicación, participación y autogestión en las instituciones educativas y en la comunidad. Para esto se planifican tareas con objetivos específicos y al finalizar se producen una síntesis y una evaluación de lo trabajado, de forma de recapitular y vivenciar los avances. El momento de cierre abarca tanto lo que acontece y se produce en el taller como los aspectos referentes a cómo se sienten participantes y coordinadores durante el mismo.

El rol del coordinador o facilitador es diferente del rol docente tradicional que enseña, en tanto la relación se torna en una tarea común de cogestión y los vínculos entre pares pasan a ser de cooperación grupal. Parte de su función es desarrollar estrategias que desplacen las miradas centradas en su persona hacia los propios integrantes del grupo para propiciar la manifestación de la interacción y la conformación de lo grupal. El facilitador promueve y crea condiciones técnicas, emocionales, grupales y comunicacionales, para que circule, 
se procese y se desarrolle el aprendizaje. Para actuar como facilitador se necesita la adquisición de herramientas tales como: el saber escuchar lo que el otro necesita, saber manejar las ansiedades y defensas que se ponen en juego en todo proceso de aprendizaje, ser tolerante, manejar la incertidumbre; se precisa conocer el contexto en el cual se va a actuar, las características de los y las participantes, las formas de acercarse y percibir la realidad que tienen. Cano (2012) enmarca el taller en la concepción metodológica de la educación popular, entendida esta como un movimiento transformador con un fin liberador organizado sobre la base de la coherencia entre fines y medios. El trabajo en taller se asume como una oportunidad pedagógica, busca dinamizar descubrimientos mediante un trabajo de análisis y problematización colectivos y concibe las contradicciones como motor del proceso dialéctico de aprendizaje.

Para la realización de los talleres se utilizan técnicas participativas, ya que las mismas son motivadoras, movilizantes, lúdicas y creativas. Admiten colectivizar el conocimiento individual, enriquecerlo y potenciar el colectivo. Las técnicas son las herramientas que hacen viable cada paso del proceso. Es decir, cuando diseñamos un taller utilizamos diversas técnicas según determinados objetivos y momentos (técnicas de caldeamiento, de presentación, para trabajar en subgrupos, para realizar una síntesis, para evaluar). Al seleccionarlas y utilizarlas debemos tomar en cuenta el contexto y coyuntura en la que se está actuando y darles "unidad estratégica" (Jara, s.f.) cuidando la coherencia interna del proceso. Según Rebellato (2009), "las técnicas son instrumentos valiosos en cuanto facilitan la dinámica del proceso grupal, crean las condiciones para una producción de conocimiento y ayudan a la emergencia de las contradicciones" (p. 52). Y advierte que "recurrimos a las técnicas que estimulan la 'participación', pero la participación no se resuelve en un complejo de técnicas y de dinámicas" (p. 52). Si su utilización es sin referencias estratégicas ni fundamentos teóricos, es posible que carezcan de la eficacia operativa para generar procesos participativos transformadores. En general, se debe adaptarlas con flexibilidad y creatividad a un grupo, situación, intereses, necesidades. Se pueden acompañar con otros recursos didácticos como la expresión corporal, plástica y musical.

\section{Metodología de trabajo}

La práctica se desarrolla en un contexto real y dinámico que provee un escenario privilegiado para la adquisición de habilidades y destrezas necesarias para el ejercicio profesional de los estudiantes en formación. Permite preparar a los futuros profesionales con una perspectiva integral, en contacto con los problemas cotidianos de las personas y participando de su resolución, desde una perspectiva ética. El curso incluye diferentes momentos; en algunos se realiza un trabajo en conjunto con la totalidad de los y las estudiantes que participan, y en otros se dividen en subgrupos para abocarse a la tarea específica asignada o elegida. Este momento inicial es de gran dificultad puesto que se deben articular las necesidades curriculares con las de la institución y con las de los y las estudiantes. Requiere de un gran esfuerzo desde el punto de vista organizativo y de coordinación del docente y flexibilidad de todas las partes. Estas dificultades son integradas desde el inicio como problemáticas a pensar y a resolver en relación con los conflictos y contradicciones que los espacios interinstitucionales y comunitarios plantean. 
Se describe brevemente a continuación el proceso que realizan los y las estudiantes de grado a lo largo del año en que cursan la práctica para ubicar la realización de los talleres dentro del mismo. Entre los meses de abril y julio efectúan un Diagnóstico de Situación Comunitario y la planificación de la intervención. ${ }^{10}$ Esta etapa de trabajo se recoge en la formulación escrita de un proyecto de intervención y un plan de actividades adecuadas a las características de los destinatarios, ajustadas según el conocimiento mutuo y la confianza que se va creando en el proceso. Entre agosto y noviembre se lleva a cabo la segunda etapa, que es la ejecución de la intervención, la evaluación y cierre. El cierre se hace con los grupos con los que se trabajó directamente y con los referentes institucionales. Este proceso forma parte de la evaluación y retroalimentación permanente, lo que da lugar a plantear la continuidad del trabajo en los años siguientes.

Los talleres fueron una de las intervenciones que se realizaron en estos años. Se ajustaron e incluyeron modificaciones fruto del aprendizaje del equipo y de los aportes de los y las participantes, tanto niños y niñas como adultos y adultas. Dentro de los objetivos, se destacaron favorecer la convivencia saludable en el ámbito escolar, promover habilidades para la vida, fomentar la autoestima y autovaloración, y estimular el sentido de pertenencia y la integración grupal.

La estructura general del ciclo de talleres comprendió un primer taller de presentación y apertura a la temática y uno final, de cierre, devolución y evaluación, y los talleres intermedios (3 ० 4) que abordaron distintos aspectos de la temática. A modo de ejemplo, en un ciclo de talleres cuya temática central fue autoestima, se trabajaron el cuidado personal, la autovaloración, el buen trato y el maltrato, la responsabilidad individual y colectiva. En algunos ciclos, la evaluación se hizo pre y posintervención, y en otros se hizo solo una evaluación posterior. La duración de cada taller fue de una hora y quince minutos.

El dispositivo de enseñanza-aprendizaje constó de tres instancias presenciales a lo largo del año lectivo: encuentros semanales de una hora y treinta minutos destinados a discutir y compartir herramientas teóricas y metodológicas, encuentros semanales de igual duración orientados a la planificación y supervisión del trabajo de campo, y el trabajo de campo propiamente dicho, de dos horas quincenales promedio desde mayo a noviembre. Los estudiantes de Psicología que participaron directamente desde 2011 a 2017 fueron 28. Otros 70 estudiantes (el resto del grupo que hizo otra intervención) compartieron y acompañaron de alguna manera la experiencia de los que trabajaron en la escuela.

En el Cuadro 1 se muestran sintetizados algunos datos de los talleres.

El Plan de Estudio de la Licenciatura en Psicología vigente (del año 2013) se organizaba a través de tres ciclos de formación. El Ciclo Inicial, con una duración de dos semestres contemplaba la introducción del estudiante a la vida universitaria y a la formación en Psicología universitaria, incorporando una perspectiva histórica e institucional de sus procesos de consolidación y desarrollo. El Ciclo Integral, de cuatro semestres de duración, tenía como objetivo brindar una formación amplia y profunda en el campo de la Psicología articulando perspectivas inter y transdisciplinares. A partir de este ciclo, el estudiante se debía involucrarse en un proyecto integral y/o una práctica preprofesional. El Ciclo de Gradua- 
ción, de dos semestres de duración, pretendía profundizar en un desarrollo académico y en prácticas que correspondieran con el interés que el estudiante definía como eje para su formación y futura labor académico-profesional.

\begin{tabular}{|c|c|c|c|c|c|}
\hline Temática & $\begin{array}{l}\text { Talleres } \\
\text { realizados }\end{array}$ & $\begin{array}{l}2 \text { Grupos/ } \\
\text { grado }\end{array}$ & Técnicas y recursos utilizados & Año/ciclo & $N^{\circ}$ estudiantes \\
\hline Vínculos & 5 & 4to. & Juegos participativos, música. & 2011 - Graduación & 5 \\
\hline Valores & 5 & 4to. & $\begin{array}{l}\text { Juegos participativos, videos, } \\
\text { imágenes, cuentos, dibujos. }\end{array}$ & 2012 - Graduación & 3 \\
\hline $\begin{array}{l}\text { Trabajo en } \\
\text { equipo }\end{array}$ & 6 & 4to. & $\begin{array}{l}\text { Collage, dramatización, técnicas } \\
\text { corporales. }\end{array}$ & 2013 - Graduación & 3 \\
\hline Pertenencia & 6 & 4to & $\begin{array}{l}\text { Sociodrama, dibujos, juego al } \\
\text { aire libre. }\end{array}$ & 2014 - Graduación & 3 \\
\hline Autoestima & 6 & 4to. & $\begin{array}{l}\text { Movimientos, técnicas de } \\
\text { relajación y representación, } \\
\text { música. }\end{array}$ & $\begin{array}{l}2015 \text { - Graduación } \\
\text { Integral }\end{array}$ & 4 \\
\hline Derechos & 6 & 5to. & Juegos tradicionales y cuentos. & $\begin{array}{l}2016 \text { - Graduación } \\
\text { Integral }\end{array}$ & 4 \\
\hline Autoestima & 6 & 5to. & $\begin{array}{l}\text { Juegos participativos, collage, } \\
\text { videos. }\end{array}$ & 2017 - Integral & 6 \\
\hline
\end{tabular}

Fuente: elaboración propia, 2020.

\section{Talleres de Promoción de Salud en una institución educativa}

Los talleres como dispositivos de trabajo con grupos pasaron a ser "un clásico" en la Escuela $N^{\circ} 171$. Si bien las maestras y la dirección de la escuela empleaban ese término en el sentido de ser algo típico o característico que se venía haciendo año a año, ciertamente nos hablaba de otra cosa. Por un lado, aludía a las expectativas e idealizaciones que la intervención producía en todos los actores del proceso: un modelo a seguir en el sentido literario, algo que "te cambia la vida" en un sentido más popular. Pero también otros sentidos adquieren visibilidad a partir de dicha nominación. Los talleres como "clásicos" parecen compartir con estos la capacidad de que cada generación los reinterprete y reinvente a su tiempo y necesidades (Kermode, 1975) o, como decía Italo Calvino (1993), "un clásico nunca agota lo que tiene para decirnos". Y de hecho el dispositivo permitía cada vez un encuentro novedoso con los niños y niñas y sus potencialidades, con los maestros y las maestras y sus esperanzas, y con los y las estudiantes de Psicología y su entusiasmo, posibilitando lo colectivo, del disfrute, del descubrimiento mutuo.

El dispositivo de trabajo en taller no enseña necesariamente algo que no se sabe sino que permite descubrirlo de otra manera, uniendo huellas y señales que están ahí para ser resignificadas. Una expresión a propósito de una actividad realizada en el patio de la escuela recoge esto: "Bueno, muchachas, dejaron su huella en la escuela". Clásico... huella... por la continuidad del trabajo, por las potencialidades del recurso y también por las repercusiones que genera en los actores que participan cada año y en toda la institución, 
siendo común que otros niños niñas y maestros y maestras pregunten por los "talleres de Psicología", "¿cuándo nos tocan?”; y en los últimos años incluso han llegado pedidos de las direcciones de otras escuelas cercanas que se enteraron de la propuesta.

Si bien se trabajó con dos grupos por año, lo que implicó en todo el período sistematizado un número aproximado de 280 niños y niñas y 14 maestros y maestras, se generó un efecto multiplicador y potenciador de la experiencia. Las vivencias que allí ocurrieron produjeron, a la hora de evaluar, una imagen muy valorada del trabajo realizado, aun atribuyéndole efectos y resultados que claramente excedían a los talleres. Esto nos inclina a pensar en aspectos que están involucrados más allá de lo concreto del trabajo. En primer lugar, se relaciona con las concepciones de sujeto y autonomía presupuestas y promovidas en el campo de la promoción de salud y la educación para la salud.

En contraposición a lo que más comúnmente encontramos en las instituciones -propuestas de trabajo y prácticas de enseñanza que se focalizan en el cambio de actitudes y hábitos, transmitido como información normativa, naturalizada e impuesta desde afuera-, en los talleres se busca problematizar, reflexionar, dar lugar a la diversidad de experiencias que portan los niños, de modo de poder integrar su cotidianeidad y facilitar la comprensión de la realidad en la que están inmersos. Recreación que posibilite cierta toma de conciencia y a partir de allí habilitar la transformación.

Otro principio promovido es el fortalecimiento de las redes sociales, la articulación interinstitucional, intersectorial e interdisciplinaria. El trabajo en red realizado en la zona, el conocimiento y la posibilidad de dar continuidad a un trabajo en conjunto de los actores articuladores (referentes de las distintas instituciones que confluyen en el territorio) de los talleres en dichas redes, hace posible un entramado que contribuye a generar un plus de efectos positivos.

Una maestra relata este proceso: "La escuela se empezó a abrir, teníamos un director que le interesaba mucho la coordinación y apareció la psicóloga que empezó a trabajar en la policlínica, otros también se abrieron". Otro maestro refiere que durante muchos años

"la escuela estaba un poquito sola, no recibía mucha gente, las únicas personas que yo vi trabajar entre 2007 y 2012 fueron estudiantes de Psicología, venir a trabajar con los niños acá, a 'embarrarse, trabajo de campo' eran los únicos... después fueron llegando otros”. (Entrevista a docente)

En este sentido, el taller vehiculiza una estrategia en la que convergen y se materializan las posibilidades de intercambios con otros de afuera de la institución, la integración de otras miradas tanto con relación a lo colectivo como a lo individual y a sostenerlos, sostenernos, acompañarlos y acompañarnos en la tarea.

Los talleres como dispositivo de trabajo permitieron lograr un espacio o momentos de ruptura en la cotidianeidad de la institución escolar que poco a poco fueron dando lugar a movimientos, algunos visibles en la institución en general y otros en los grupos, maestros y maestras con los que se trabajó. Ruptura en lo espacial, en lo pedagógico, en lo vincular, en la concepción del rol del psicólogo. Iremos señalando cómo las propuestas, ideas y concepciones de trabajo se fueron incorporando y tomando cuerpo en la institución, sin desconocer la incidencia de otros factores y actores en estos cambios ni confundiendo formas con contenidos - no por sentarnos en círculo promovemos autonomía. 
Desde que empezamos a trabajar en la escuela se observaron propuestas individuales, puntuales y aisladas, de maestras que buscaban generar cambios en el modelo tradicional de enseñanza, pero estas no alcanzaban la consolidación suficiente como para transformarlas en fuerzas instituyentes (Lourau, 2001). Seguramente un nivel de explicación válido, entre otros, sea que las maestras y los maestros no cuentan en su formación con las herramientas para sostener y abordar los emergentes que este tipo de cambios puede habilitar ni cuentan con el tiempo suficiente para hacerlo. Tal vez tampoco los grupos tengan la madurez suficiente como para actuar en forma independiente del modelo instituido. Nuestra intervención sumó en ese sentido al mostrar una metodología que rompía con representaciones y prácticas internalizadas, propias de un modelo que mantenía las características de la educación "bancaria", con la tradicional hilera de bancos mirando al pizarrón y el maestro adelante, como figura de autoridad cuyo rol es transmitir e ilustrar a los alumnos, carentes de saber. Se pusieron en suspenso, al menos momentáneamente, los viejos roles y se habilitaron nuevas lógicas en los procesos de enseñanza-aprendizaje. Se movieron bancos, se trabajó en el suelo y en el patio, con música y murmullos. La disposición diferente permitió que circularan de manera distinta los saberes, roles y jerarquías. Todos podían observarse mutuamente y ser vistos-atendidos por todos, aunque se fue muy flexible en la modalidad de participación de cada uno y cada una, considerando las singularidades. Se partió de la vida cotidiana, incluyendo los intereses de los niños y las niñas, su afectividad, favoreciendo que asumieran un rol activo. Jugando, dialogando, escuchando al compañero, creando juntos, disfrutaron y aprendieron sobre diferentes temáticas casi sin darse cuenta.

Lo que sucedía en los talleres comenzó a circular en toda la institución a la vez que se fue difundiendo entre las familias de los niños que participaban de los talleres, de manera que se expandió entre las redes cercanas. Es sabido que los roles se aprenden y su aprendizaje requiere deconstrucción y reconstrucción tanto para los niños como para los adultos. En ese aspecto, acordamos trabajar en temáticas tales como autoestima, derechos, pertenencia, integración grupal, es decir, se jerarquizó el trabajo en y sobre los vínculos —consigo mismo, con los otros, pares y adultos, con la institución-. La propuesta generó movilizaciones y repercusiones en los y las participantes y en las prácticas institucionales. Los niños y las niñas, en la mayoría de los casos, se involucraron, algunos más, otros menos, algunos colaborando con la tarea, otros dificultándola, algunos desde el principio, otros hacia el final. En algunos talleres relataron sus vivencias abiertamente, incluso de experiencias difíciles; en otros participaron desde el "deber ser", dejando afuera las emociones. Para todos fue una experiencia diferente cuyos efectos se empezarían a ver con el tiempo.

El mayor logro de los talleres, a nuestro entender, está relacionado con la ruptura y el cambio en la mirada que se logra instaurar en los y las docentes. En el trabajo con los niños salen a la luz potencialidades y recursos que sorprenden a maestras, compañeros y compañeras. También sucede que algunos de los niños que habitualmente tienen problemas de conducta, "los que no se quedan quietos, o no dejan trabajar a los demás", presentan cambios en su actitud, plegándose al trabajo. Año tras año confirmamos el efecto del cambio de la mirada sobre los niños, las niñas y el grupo. La mirada fresca, bien dispuesta y habilitante, de los estudiantes de Psicología, operó en la mirada de maestros y maestras, de sí mismos y del grupo de pares. Se confirmó que si el adulto referente se posiciona desde las potencialidades del niño y de la niña en lugar de en las carencias o dificultades, esto 
se traduce en una ganancia en cuanto a la confianza y autoestima de ellos y ellas. Estos logran visualizar sus propias capacidades y las de sus compañeros, lo cual puede favorecer el intercambio de roles en lo grupal, generando movimientos. Para la maestra, este cambio de mirada igualmente implica romper con la vivencia de frustración e impotencia expresada en términos de "la situación es difícil, ni a los padres, ni a los niños le interesa la escuela o aprender", "es muy desgastante", por una mirada de esperanza en sus propias capacidades para lograr enseñar y en las capacidades de los niños y las niñas para aprender. Los niños dicen cosas como: "me gustó que estuviéramos todos juntos jugando y aprendiendo", "que vengan a trabajar con nosotros". A su vez, se producen efectos en cuanto a vivir la escuela como un lugar agradable, cambiando posibles vivencias respecto de la misma, afectando los sentimientos de pertenencia institucional.

Respecto de los maestros, en las entrevistas que se realizaron los primeros años de la práctica surgió en forma reiterada el pedido de ayuda frente a niños problemáticos, conflictivos, nos contaron sus "historiales" y demandaron el rol asistencial tradicional del psicólogo. Se dieron incluso situaciones en que estas entrevistas, cuyo objetivo fue conocer la institución o los diferentes grupos, funcionaron como espacio de catarsis donde se relató todo tipo de experiencias traumáticas que atravesaban los niños, las niñas, los maestros y las maestras, dado que estos se sentían francamente desbordados y el manejo para los y las estudiantes resultaba muy difícil. A partir de la búsqueda del equilibrio necesario entre el rol asignado y el asumido, paulatinamente esto fue cediendo en cuanto a la comprensión del encuadre y los objetivos de la intervención y a la posibilidad de acercamiento a la problemática desde otras concepciones que incluían las dimensiones sociohistóricas, políticas y económicas (Paradigma de la Complejidad, Morin, 1990) en lugar de una concepción reduccionista, estática e individualista de la realidad (Paradigma Clásico). También fue factible dar visibilidad a la sobrecarga de funciones que "desbordaba" el rol pedagógico clásico para el cual habían sido formados maestros y maestras y los efectos que esto causa en ellos y en su trabajo, por lo que se propusieron espacios para trabajar grupalmente. Propuesta que, en principio, no prosperó, pero en los últimos años se pudo hacer algún movimiento en ese sentido. Se logró incorporar a sus espacios de trabajo en conjunto instancias puntuales como, por ejemplo, el cuidado del cuidador.

Con referencia a las maestras y los maestros y su participación en los talleres, hubo diversidad de respuestas. Encontramos resistencias pasivas a la dinámica de trabajo, lo que generó al inicio situaciones de ansiedad frente al cambio de roles, lugares y temáticas a trabajar. Muchos çse sintieron interpelados, no encontraron su lugar en el dispositivo y permanecieron por fuera del mismo; otras y otros actuaron desde su rol tradicional, interfiriendo más allá de lo deseable en la propuesta de trabajo. También hubo quienes apoyaron la tarea, con apertura y entusiasmo, participaron en más de un año y reconocieron las carencias propias y del sistema.

"Lo que trabajen con ustedes no lo van a hacer o a aprender en otro lugar, no tienen la posibilidad, hay un mundo que ustedes les van a acercar... son otros contenidos que ellos necesitan y a nosotros no nos da el tiempo para abordar esos temas, a mí me encantaría... estoy aprendiendo con ustedes". (Entrevista con docente) 
La continuidad de la presencia en la institución, el trabajo en red, la articulación interinstitucional, intersectorial e interdisciplinaria en conjunto en el territorio favoreció la instalación de un vínculo de confianza que permitió mejorar y profundizar la propuesta integrándose poco a poco maestros y maestras que al inicio no estaban dispuestos a participar. Asimismo, se logró superar, en parte, las fantasías y miedos con respecto a qué haríamos los psicólogos en la institución. Comenzó a primar el sentirse acompañados por un otrotercero en el aula, sostenidos interdisciplinariamente en aquello que los desbordaba. Desde ahí algunos y algunas pueden ubicarse en otro lugar, cambiar la mirada, la perspectiva. Sin embargo, ciertos comentarios que recibimos tanto de los niños y niñas como de maestros y maestras dan cuenta también de las dificultades de significar y valorar la experiencia. Un niño preguntó: "Hasta qué hora están ustedes?". "Hasta las 12" — se le respondió. "Bien ahí, mejor, así no hacemos nada — dijo él. Una maestra comentó al retirarse los estudiantes: "Espero que les esté sirviendo".

Los estudiantes de Psicología que participaron tomaron contacto con la realidad de otras personas y sus problemas, adecuando expectativas y fantasías con relación al otro, a sí mismos y a la disciplina. Tuvieron la posibilidad de articular y poner en juego los conocimientos teóricos adquiridos a lo largo de la formación y de mejorar sus habilidades comunicacionales y la confianza en sí mismos y en su capacidad de aportar a otros. La participación es otro factor que contribuyó a dar un plus a la experiencia ya que su juventud fue un facilitador para la comunicación y un atractivo que generó curiosidad e impacto en los niños y las niñas: alguien de afuera del barrio que iba a trabajar y a aprender con ellos, que les proponía otras formas de aprender, otra disposición. En una amplia mayoría, lograron establecer vínculos muy adecuados y empáticos con los niños, niñas (en especial), maestras y maestros con los cuales trabajaron. Algunos quedaron muy sensibilizados con la realidad con la que tomaron contacto y sintieron impotencia frente a situaciones que excedían los objetivos de la intervención. Desde el punto de vista formativo, demostró ser una propuesta pedagógica que los desafió en varios sentidos. Se destaca la necesaria flexibilidad para adaptarse a los imprevistos y cambios que necesariamente fueron surgiendo sobre la marcha. La formación de equipos de trabajo es otra tarea que debieron ir haciendo a la par del proceso de intervención. Los y las estudiantes no se conocían entre sí y se debían poner a trabajar juntos para desarrollar progresivamente su capacidad de relacionarse y autogestionarse. El trabajo de equipo no es sin conflictos así como tampoco lo es la inserción en una institución escolar atravesada por otras lógicas de funcionamiento, además de las ansiedades propias del nuevo rol. Sin embargo, podríamos decir, que salvo alguna excepción, las dificultades que surgieron a la interna de los equipos se fueron resolviendo en forma adecuada y en los tiempos necesarios para poder asimilarlos y seguir adelante con la tarea. Los y las estudiantes de Psicología, como la mayoría de las personas, tienen un conjunto de ideas previas, fantasías y expectativas con respecto a lo que es un taller, cómo se trabaja o si sirve para algo. Sorprende encontrarse con que el término es mucho más usado que comprendido, se le llama taller a distintas cosas, desde instancias de recreación o entretenimiento hasta una charla informativa. Si bien podría pensarse que los y las estudiantes pueden estar más formados o tener desarrollada algunas de las habilidades que el taller requiere, esto no se constata en la mayoría de los casos. Al llegar al curso, los estudiantes carecen de una mirada estratégica para la planificación con la cual utilizar las técnicas participativas y corren el riesgo de reali- 
zar una práctica meramente "espontaneísta o voluntarista" (Cano, 2012) donde se hace "por hacer", se llena un espacio, sin entender los objetivos. El aprendizaje de la metodología de trabajo en taller requiere una larga y cuidadosa preparación y planeación para alcanzar los objetivos que posibiliten la toma de conciencia y las transformaciones. Otro aspecto importante en el que es necesario trabajar es el rol del coordinador, sujeto que enseña de manera no tradicional, que guía y conduce un proceso, teniendo en cuenta las opiniones, vivencias, diferencias y ritmos de los y las participantes. Los temas se trabajan, se discuten, se retoman, se ven desde distintos puntos de vista, se reconducen, se sintetizan. El coordinador tiene que potenciar la participación y valorar el saber de todos, tiene que realizar una redistribución del saber-poder y este rol no está dado de antemano, requiere que sea construido. El espacio del taller surge una y otra vez en los trabajos de reflexión final de los estudiantes como un dispositivo a incorporar en las escuelas tanto para trabajar con niños y niñas como con adultos y adultas. Resaltan la posibilidad de dar cabida a lo singular y a lo diverso, a sostener y responder a múltiples realidades sin reducirlas ni encasillarlas, a que los conflictos se transformen en diálogo y aprendizaje. Los maestros, las maestras, los niños y las niñas también lo mencionaron: "Necesitaríamos estas instancias con más frecuencia", "que sigan viniendo a trabajar con nosotros", "continuar con el trabajo en modalidad de talleres ya que los niños lo disfrutan y aprovechan". Resaltaron las actividades que se proponían donde tenían que participar activamente: "Que vengan todos los años", "trabajar con ustedes en todas las cosas".

\section{Reflexiones finales}

La experiencia relatada supone la articulación de recursos e implementación de diferentes estrategias para el trabajo en el PNA y la promoción de salud que dieron como resultado un aporte creativo e innovador para la zona. En ese momento y condiciones, fue posible articular algunas de las demandas de la comunidad con las necesidades de formación de recursos humanos a nivel universitario para el trabajo en el PNA. Destacamos, por ejemplo, la participación de diversos actores que confluyeron en un territorio, la posibilidad de un proceso de enseñanza-aprendizaje alrededor de problemas reales y cotidianos de una comunidad, el trabajo en el caso de los niños y niñas con referentes adultos más cercanos en edad, la factibilidad de crear y realizar un plan de intervención a medida, la introducción de herramientas de trabajo y temáticas no incluidas tradicionalmente en la educación formal. Es decir, se dio un escenario en el que fue posible introducir aspectos que resultaron novedosos en la cotidianeidad de la institución educativa, ya que se estimularon lo grupal, las interacciones diferentes entre los participantes, con mayor libertad, el trabajo colaborativo, se incluyeron los afectos, lo lúdico y la creatividad. La permanencia en el tiempo y el pedido de seguir con la experiencia demuestran sus efectos positivos, transformadores y potenciadores en los niños, las niñas, los adultos y adultas que forman parte de la misma. Se acompaña y sostiene, aportando otra mirada en la institución educativa. Aumenta el conocimiento de los aportes de la Psicología en el PNA, la imagen de algunos niños y niñas cambia e incluso sorprende a los maestros y las maestras al ver el despliegue de una potencialidad oculta. Para los y las estudiantes de Psicología es una forma de interrogarse a sí mismos acerca de sus posibilidades y competencias para el futuro rol profesional. 


\section{Referencias bibliográficas}

Ander Egg, E. (1991). El taller una alternativa de renovación pedagógica. Magisterio del Río de la Plata.

Calvino, I. (1993). Por qué leer los clásicos. Tusquets.

Cano, A. (2012). La metodología de taller en los procesos de educación popular. ReLMeCS, 2(2, julio-diciembre), 22-52.

CEIP (2014). Programa APRENDER, Haciendo memorias. Consejero Héctor Florit. CEIP.

Cortazar, M.; Rydel, D.; Gandolfi, A (2009). La Herramienta de Diagnóstico de Situación Comunitario. 1er. Congreso Universitario de diagnóstico e Intervención Psicológica Caleidoscopio de Prácticas. Facultad Psicología, UdelaR.

Cortazar, M.; Gandolfi, A.; Rydel, D. (2010). Construyendo modelos de intervención desde la Psicología de la Salud. Publicación de la Sociedad Uruguaya de Psicología Médica y Medicina Psicosocial. SUPM

Freud, S. (1924). El sepultamiento del complejo de Edipo. Obras completas, 19. Amorrotu.

Giorgi, V. (s.f.). A propósito del trabajo de los equipos de salud con la comunidad. Problemas y potencialidades.

(2006). Construcción de la subjetividad en la exclusión. En Encare (Comp.), Drogas y Exclusión Social (pp. 10-11). Atlántica.

Gonzalez, M.T. (1987). El taller de los talleres. Ángel Estrada.

Jara, O. (s.f.). La concepción metodológica dialéctica, los métodos y las técnicas participativas en la educación popular. Centro de Estudios y Publicaciones Alforja.

Kermode, F. (1975). El clásico: imágenes literarias de permanencia y cambio. Nueva York, Viking Press, ISBN 0-670-22508-8, OCLC 1207405.

Lourau, R. (2001). El análisis institucional. Amorrortu.

Montero, M. (2004). Introducción a la psicología comunitaria. Desarrollo, conceptos y procesos. Paidós.

(2006). Teoría y práctica de la Psicología Comunitaria. Paidós.

Morin, E. (1990). Introducción al Pensamiento Complejo. Gedisa.

Organización Mundial de la Salud (OMS) (1986). Carta de Ottawa para la Promoción de Salud. Primera Conferencia Internacional sobre Promoción de Salud: hacia un nuevo concepto de Salud Pública. OMS Canadá.

Organización Panamericana de la Salud (OPS) (1999). Planificación Local Participativa. OPS/OMS.

Piaget, J. (1968). Psicología de la Inteligencia. Proteo.

Rebellato, J. L. (2009). Educación popular y cultura popular. En Brenes, A.; Burgueño, M.; Casas, A. \& Pérez, E. (Comps.). José Luis Rebellato. Intelectual radical. Extensión Libros, Nordan Comunidad \& EPPAL.

Rydel, D. (2014). Intervenciones en el primer nivel de atención en salud. Poster. Trabajo de cierre de curso de Prácticas y Proyectos / Ciclo de Graduación. Feria de Salud, Empalme Nicolich.

Terigi, F. (2009). Las trayectorias escolares. Del problema individual al desafío de política educativa. Proyecto Hemisférico Elaboración de políticas y estrategias para la prevención del fracaso escolar. OEA y Agencia Interamericana para la Cooperación y el Desarrollo (AICD).

Urribarri, R. (1999). Descorriendo el velo. Sobre el trabajo de latencia. Revista Latinoamericana de Psicoanálisis, 3(1), 257-292. FEPAL.

Valdés, R. y Klasse, E. (2008). Ayudemos al niño a aprender a convivir. Guía facilitadora para los maestros comunitarios \#3. UNICEF. 